Article

\title{
MADS-Box Transcription Factor SsMADS Is Involved in Regulating Growth and Virulence in Sclerotinia sclerotiorum
}

Xiaoyan Qu ${ }^{1, \dagger}$, Baodong Yu ${ }^{2, \dagger}$, Jinliang Liu ${ }^{1}$, Xianghui Zhang ${ }^{1}$, Guihua Li ${ }^{1}$, Dongjing Zhang ${ }^{1}$, Le Li ${ }^{1}$, Xueliang Wang ${ }^{1}$, Lu Wang ${ }^{1}$, Jingyuan Chen ${ }^{1}$, Wenhui Mu ${ }^{1}$, Hongyu Pan ${ }^{1, *}$ and Yanhua Zhang ${ }^{1, *}$

1 College of Plant Science, Jilin University, Changchun 130062, China;

E-Mails: xiaoyanQu1988@163.com (X.Q.); jlliu@jlu.edu.cn (J.L.); zhangxianghui@jlu.edu.cn (X.Z.); liguihua@jlu.edu.cn (G.L.); m186266180402@163.com (D.Z.); lile19890904@126.com (L.L.); mewxl@163.com (X.W.); believewanglu@126.com (L.W.); jingyuanchen90@gmail.com (J.C.); muwenhui2004@163.com (W.M.)

2 Department of Emergency, China-Japan Union Hospital, Jilin University, Changchun 130033, China; E-Mail: ybd@jlu.edu.cn

$\dagger$ These authors contributed equally to this work.

* Authors to whom correspondence should be addressed;

E-Mails: panhongyu@jlu.edu.cn (H.P.); yh_zhang@jlu.edu.cn (Y.Z.);

Tel.: +86-431-8783-5712 (H.P. \& Y.Z.); Fax: +86-431-8783-5659 (H.P. \& Y.Z.).

Received: 17 March 2014; in revised form: 10 April 2014 / Accepted: 17 April 2014 /

Published: 8 May 2014

\begin{abstract}
MADS-box proteins, a well-conserved family of transcription factors in eukaryotic organisms, specifically regulate a wide range of cellular functions, including primary metabolism, cell cycle, and cell identity. However, little is known about roles of the MADS-box protein family in the fungal pathogen Sclerotinia sclerotiorum. In this research, the $S$. sclerotiorum MADS-box gene SSMADS was cloned; it encodes a protein that is highly similar to Mcm1 orthologs from Saccharomyces cerevisiae and other fungi, and includes a highly conserved DNA-binding domain. MADS is a member of the MADS box protein SRF (serum response factor) lineage. SSMADS function was investigated using RNA interference. Silenced strains were obtained using genetic transformation of the RNA interference vectors pS1-SsMADS and pSD-SSMADS. SsMADS expression levels in silenced strains were analyzed using RT-PCR. The results showed that SsMADS mRNA expression in these silenced strains was reduced to different degrees, and growth rate in
\end{abstract}


these silenced strains was significantly decreased. Infecting tomato leaflets with silenced strains indicated that SsMADS was required for leaf pathogenesis in a susceptible host. Our results suggest that the MADS-box transcription factor SsMADS is involved in S. sclerotiorum growth and virulence.

Keywords: Sclerotinia sclerotiorum; RNA interference; transcription factor; MADS-box

\section{Introduction}

Sclerotinia sclerotiorum (Lib.) de Bary is a notorious necrotrophic fungal pathogen with an extensive host range, endangering 278 families and more than 450 types of plants [1]. Plant sclerotinia is caused by the fungus $S$. sclerotiorum, which is an important cause of disease worldwide; this fungus mainly affects rape, sunflowers, soybeans and other oilseeds, lettuce, carrots, and other vegetable crops. The incidence of sclerotinia, in susceptible areas is up to $80 \%$, particularly in oilseed rape. Rapeseed flu disease usually further reduces yield by $10 \%$ to $70 \%$, and sclerotinia reduces the oil content by $1 \%$ to $5 \%$, resulting in huge economic losses $[2,3]$.

Our group constructed a $S$. sclerotiorum apothecia cDNA library using SMART (Switching Mechanism at 5' end of RNA Transcript). EST (expressed sequence tags) sequences were obtained by measuring and analyzing the $S$. sclerotiorum apothecia cDNA library. There were 242 genes identified using bioinformatic analysis, and we obtained a gene that is highly similar to Mcml orthologs from Saccharomyces cerevisiae, Magnaporthe oryzea, and other fungi, including a MADS-box highly conserved DNA-binding domain. This gene was called SsMADS (GenBank accession no. FJ869956).

MADS-box proteins are a family of transcription factors that are well conserved in eukaryotic organisms including yeasts, plants, insects, amphibians, and mammals, and are involved in the regulation of a vast range of cellular functions through interaction with their cofactors $[4,5]$. The MADS-box proteins have a highly conserved sequence motif that was identified after sequence comparison with MCM1 (yeast), Agamous (plant), Deficiens (Drosophila), and SRF (serum response factor, human) [3]. Its founding members are MADS-box proteins that are classified into two subfamilies: the SRF-like subfamily (type I) and MEF2 (myocyte enhancer factor 2)-like subfamily (type II), based on the amino acid sequence of the conserved MADS-box [3]. In plants, MADS-box proteins are a flourishing family in flowering plants and contain several MADS-box genes. Arabidopsis has been shown to contain many MADS-box proteins, with 107 members have been identified in this species [6]. Studies have shown that, at different stages of plant growth and development (such as the seedling, and flowering stages), different parts (such as roots, stems, leaves, flowers, fruits, and seeds) have varying degrees of MADS-box expression, and that MADS-box play an important regulatory role [2,7-9].

Only a few MADS-box genes are present in animals. SRF-like MADS box factors in animals mediate the signal responsive transcription of immediate-early genes such as c-fos, actin gene, and junB [10]. SRF also regulates muscle-specific genes, which are expressed in post-mitotic muscle cells [11]. In mammalian cells, there are four MEF2 genes, mef $2 \mathrm{~A}$, mef $2 \mathrm{~B}$, mef $2 \mathrm{C}$, and mef $2 \mathrm{D}$, which are expressed in distinct patterns during embryogenesis and in adult tissues [12]. Taking advantage of the 
increasing number of whole genome sequences and the progress in bioinformatics tools, research on MADS-box genes is also progressing.

Related MADS-box transcription factors have been reported in fungi. MCM1 is central to the transcriptional control of cell-type specific genes and the pheromone response in S. cerevisiae [4]. However, to date, MADS-box protein in S. sclerotiorum has not been reported. To further understand the biological role of MADS-box transcription factors in the development and pathogenesis of S. sclerotiorum, in this study, the SSMADS gene was cloned and its function was investigated using RNA interference technology.

\section{Results and Discussion}

\subsection{S. sclerotiorum SsMADS Is a SRF (Serum Response Factor)-MADS-Box Protein}

We isolated the putative MADS-box transcription factor SSMADS from S. sclerotiorum, and investigating the known fungal MADS-box transcription factors. Similar to other filamentous ascomycetes, such as Neurospora crassa, M. oryzae, Aspergillus nidulans, and Fusarium verticillioides, S. sclerotiorum has two putative MADS-box transcription factors (SS1G_05588 and FJ869956). One of MADS-box transcription factor (accession no. FJ869956) is called SsMADS. The SsMADS gene length is $847 \mathrm{bp}$, which encodes a putative protein that is 226 amino acids long and has two introns of 106 and $61 \mathrm{bp}$ in length. The predicted S. sclerotiorum SSMADS gene has 86\%, 82\%, and 71\% homology with the respective nucleic acid exons sequences of Aspergillus oryzae (Mcm1), A. nidulans (MCMA) and M. oryzae (MoMCM) [13]. Phylogenetic analysis indicated that S. sclerotiorum SSMADS, F. graminearum FGSG 08696, M. oryzae MoMCM1, N. crassa NCU07430, A. nidulans $M C M A$, and P. digitatum PDIP 61540 belong to the SRF-like clade represented by S. cerevisiae Mcm 1 (Figure 1A). The A. nidulans RlmA, N. crassa NCU02558, O. piceae F50303505, M. oryzae Mig1, F. graminearum FGSG 09339, and S. cerevisiae Rlm transcription factor genes are conserved in filamentous ascomycetes belonging to the MEF2-like class (Figure 1A). This domain within S. sclerotiorum SsMADS exhibited a high degree of sequence identity with the MADS-box domains of the four MADS-box family founder proteins (MCM1, Agamous, Deficiens, and SRF) of eukaryotic transcriptional regulators (Figure 2B). The putative S. sclerotiorum SsMADS protein sequence was submitted to the MADS SRF-like/Type I subfamily, and the presence of the SRF-like MADS-box domain (amino acids 57 to 136), a distinct DNA-binding motif, was recorded [14]. Based on the sequence homology within the domain adjacent to the MADS-box and phylogenetic analysis, S. sclerotiorum SsMADS is a member of the MADS-box protein SRF lineage.

\subsection{The RNA Silencing Vectors $p S 1-S s M A D S$ and $p S D-S S M A D S$ Constructed}

To assess the role of SsMADS in S. sclerotiorum, we constructed S. sclerotiorum SsMADS silent strains using RNA interference. For this purpose, the respective 520 bp SsMADS gene fragment was cloned, and the digested products were ligated into pSilent-1 and pSilent-Dual1, thus, constructing two SsMADS RNA-silencing vectors pS1-SsMADS and pSD-SsMADS (Figure 2). The two RNA-silencing vectors can be used in subsequent transformant studies. 
Figure 1. Phylogenetic analysis of SsMADS and alignment with related proteins. (A) Phylogenetic analysis of S. sclerotiorum SsMADS and other MADS-box transcription factors from $S$. cerevisiae, F. graminearum, M. oryzae, N. crassa, P. digitatum, and O. piceae, showing evolutionary relationships of fungal SRF (serum response factor)-like and MEF (myocyte enhancer factor)-like MADS-box proteins. Phylogenies were speculated using MEGA (version 5.05) to create an unrooted phylogenetic treeand; (B) MADS box domains of $S$. sclerotiorum SsMADS, S. cerevisiae Mcm1p (ScMCM1, accession no. CAA88409.1), human SRF (HsSRF, accession no. CAI13785), A. thaliana AGAMOUS (AtAG, accession no. P17839), and Antirrhinum majus DEFICIENS (AmDEF, accession no. CAA44629), aligned to maximize similarities using DNAMAN (version 6.0.3).

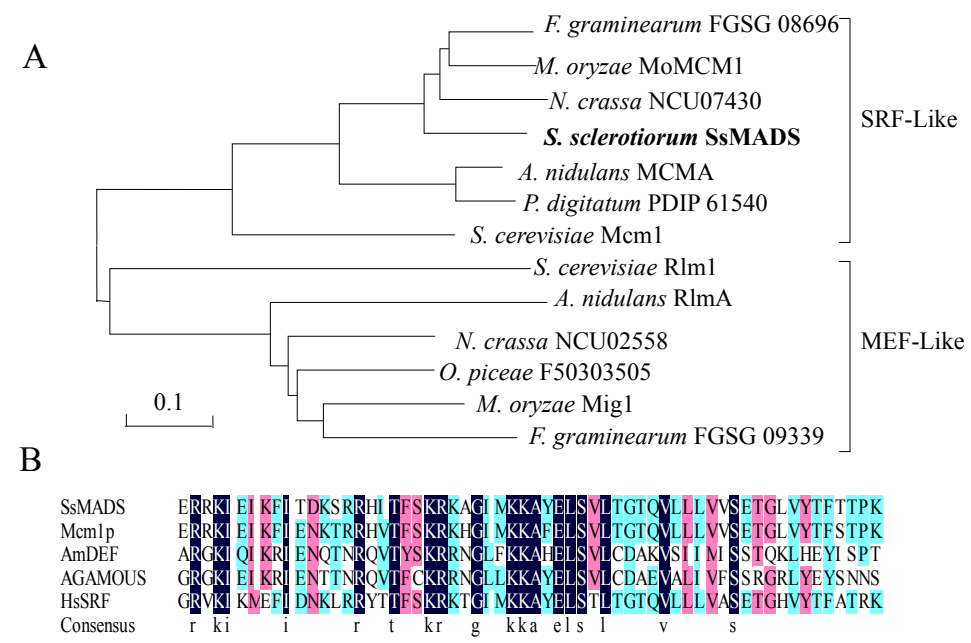

Figure 2. Restriction maps of the $S S M A D S$ RNAi vector. (A) Original pSilent-1 vector map [15]; (B) Two 520 bp fragments of the SsMADS gene were amplified from cDNA of S. sclerotiorum and inserted into the pSilent-1 vector at the XhoI, HindIII and BglII, and KpnI sites, resulting in pS1-SsMADS; (C) The original map of the pSilent-Duall vector [16]; and (D) A $520 \mathrm{bp}$ fragment of the $S s M A D S$ gene was amplified from cDNA of $S$. sclerotiorum and inserted into the pSilent-Duall vector at the XbaI site, resulting in $\mathrm{pSD}-S S M A D S$.

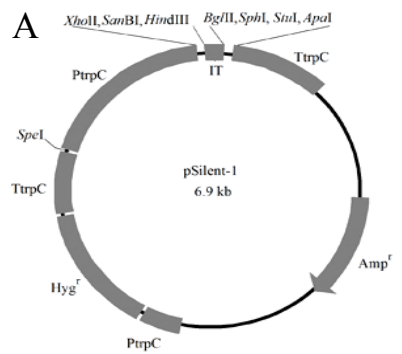

C Xbal,Spel, BamHI, Smal, Pstl, EcoRI, EcoRV, HindIII

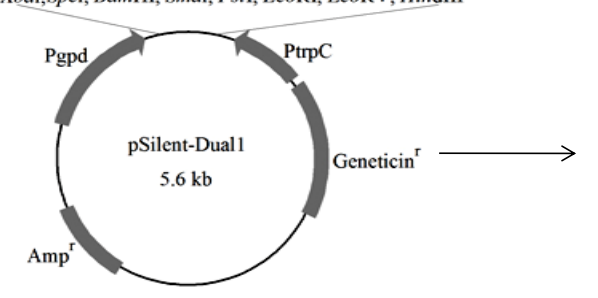

B

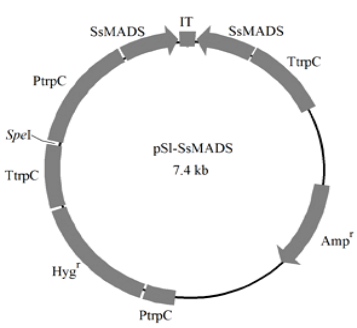

$\mathrm{D}$

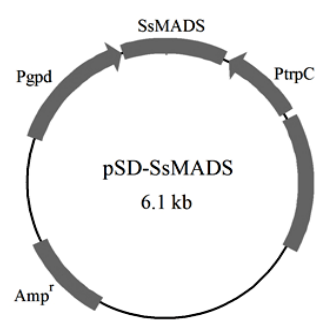




\subsection{The Acquisition of RNAi-Positive Transformants}

To determine the function of SsMADS gene in S. sclerotiorum, the silencing vectors pS1-SsMADS and pSD-SsMADS were constructed by inserting the SSMADS fragment into apSilent-1 and pSilent-Dual1 plasmid. The reconstructed plasmids were transformed into protoplasts of wild-type S. sclerotiorum 1980 strain. In this study, S. sclerotiorum protoplasts as receptors to facilitate introduction of exogenous genes. The transformation efficiency was approximately1 in every $6.0 \times 10^{4}$ S. sclerotiorum protoplasts that had a transformant introduced. Approximately 200 transformants were screened by adding $100 \mu \mathrm{g} / \mathrm{mL}$ hygromycin B or geneticin in PDA medium. Ten putative SSMADS-silencing transformants, $\mathrm{pS1} 1$, $\mathrm{pS1} 1-2, \mathrm{pS} 1-3, \mathrm{pS} 1-4$, and $\mathrm{pS1-5}$ (from reconstructed plasmid pS1-SSMADS transformations) and pSD-1, pSD-2, pSD-3, pSD-4, and pSD-5 (from reconstructed plasmid pSD-SSMADS transformations), had typical phenotype changes (Figure 3). These transformations were identified as positive using amplifying hygromycin B phosphotransferase or geneticin-resistant genes with their specific primers (hyg-F/hyg-R or gen-F/gen-R). In transformants pS1-1, pS1-2, pS1-3, pS1-4, pS1-5, an 810 bp specific fragment of the hygromycin $B$ phosphotransferase gene was amplified, and in transformants pSD-1, pSD-2, pSD-3, pSD-4, and pSD-5, an 537 bp specific fragment of geneticin-resistant gene was amplified (Figure 4A,B). The $S S M A D S$ expression level was analyzed using real-time RT-PCR. Transformant pSl-1 SsMADS expression level was slightly increased compared to the wild-type. The other SsMADS transformants' expression was significantly reduced compared to wild type, but there were differences in the degree of the decrease: transformants pSD-1 and pSD-3 slightly decreased compared to wild type, while pS1-2, pSl-3, pSl-4, pS1-5, pSD-2, pSD-4, and pSD-5 expression in the SsMADS was significantly lower than the wild type. These results showed that SsMADS mRNA expression in the transformants was reduced to different degrees.

Figure 3. Colonial morphology of wild type and transformant strains after incubation on PDA medium for 10 days at $25^{\circ} \mathrm{C}$. Strain W is the wild-type strain, S. sclerotiorum 1980. Strains pS1-1 to pS1-5 are from reconstructed plasmid pS1-SSMADS transformation and strains pSD-1 to pSD-5 are from reconstructed plasmid pSD-SSMADS transformation.

W

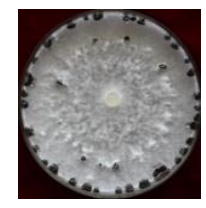

W

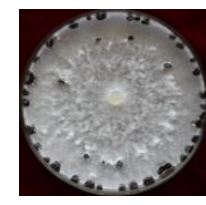

pS1-1

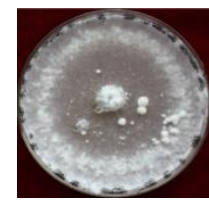

pSD-1

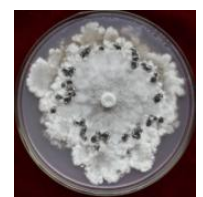

pS1-2
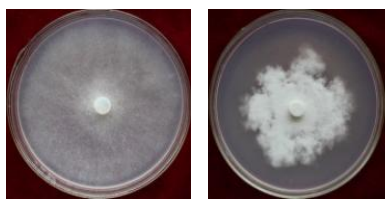

pSD-2

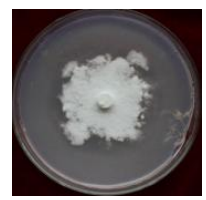

pSD-3

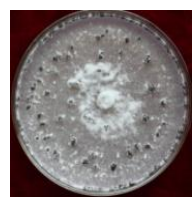

pS1-4
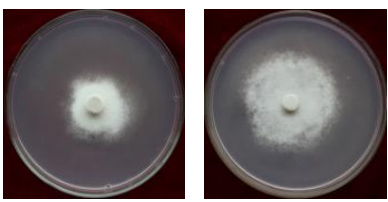

pSD-4

pSD-5

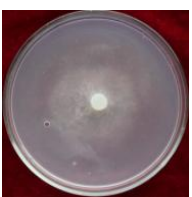


Figure 4. Silencing status of $S$. sclerotiorum SSMADS. (A) Wild-type strain and transformants identified by amplifying the hygromycin gene fragment using specific primers (hyg-F/hyg-R). M: DNA molecular size marker DL2000; W: wild type strain; lanes 1-5: putative transformants pSl-1 to pSl-5; (B) Wild-type strain and transformants identified by amplifying geneticin-resistant gene fragment using specific primers (gen-F/gen-R). M: DNA molecular size marker DL2000; W: wild-type strain; lanes 1-5: putative transformants pSD-1 to pSD-5; (C,D) SSMADS expression level in wild type (WT) and transformant strains pSl-1 to pS1-5 and pSD-1 to pSD-5 were determined using real-time RT-PCR. The SSMADS cDNA expression level was normalized to that of $\beta$-actin cDNA in extracts from each strain. The abundance of cDNA from the wild type was assigned a value of 1 . Bars indicate standard error.

A

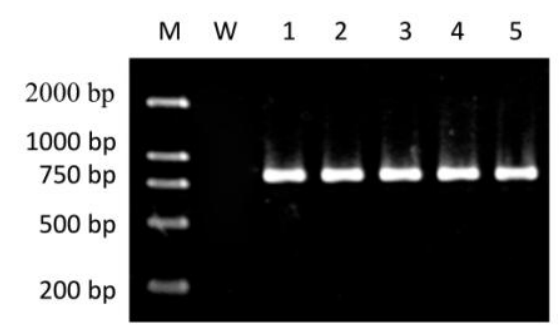

$\mathrm{C}$

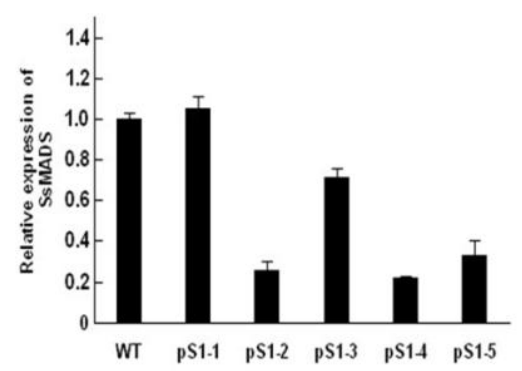

B

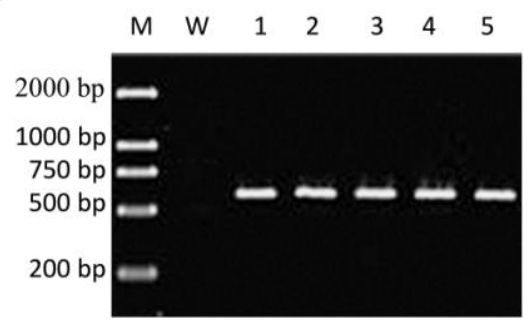

D

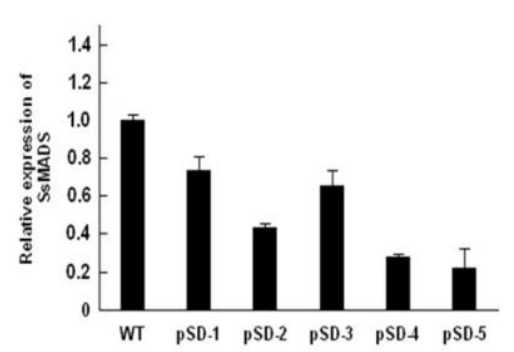

\subsection{Silencing of SsMADS Reduced the Aerial Hyphal Growth Rate}

These mutants were phenotypically indistinguishable using colony morphology. To determine the role of SSMADS in S. sclerotiorum hyphal growth, we compared the aerial hyphal growth rate of the wild-type strain and SsMADS-silencing transformants pS1-1, pS1-2, pS1-3, pS1-4, pS1-5, pSD-1, pSD-2, pSD-3, pSD-4, and pSD-5. Colony diameter of the wild-type strain, S. sclerotiorum 1980, and transformant strains cultured on PDA medium was measured to show the growth rate of the hyphae. SSMADS-silencing strains pSl-1, pSD-1 and pSD-3 had a similar growth rate to wild type. However, the other SSMADS-silencing strains pSl-2, pSl-3, pSl-4, pSl-5, pSD-2, pSD-4, and pSD-5 had a significantly slower growth rate compared with the wild-type (Figure 5A,B). Transformants pS1-3 and pSD-3 SSMADS expression level lower than wild-type strain, but higher than the transformants pS1-2, pS1-4, pS1-5 and pSD-2, pSD-4, pSD-5 (Figure 4C,D), and their aerial hyphal growth rate also lower than wild-type strain, but higher than transformants pS1-2, pS1-4, pS1-5 and pSD-2, pSD-4, pSD-5 (Figure 5A,B). There were significant differences in the hyphae growth rate between the wild-type strain and transformants $(p<0.01)$. These results suggest that SSMADS plays a major role in aerial hyphal growth. 
Figure 5. Determination of wild-type and radial growth in SSMADS-silencing transformant strains cultivated on PDA solid medium and incubated for 2 days at $25{ }^{\circ} \mathrm{C}$. (A) Radial growth determination of the wild type strain (W) and the transformants pSl-1 to pSl-5; (B) Radial growth determination of the wild type strain (W) and transformants pSD-1 to pSD-5.

A

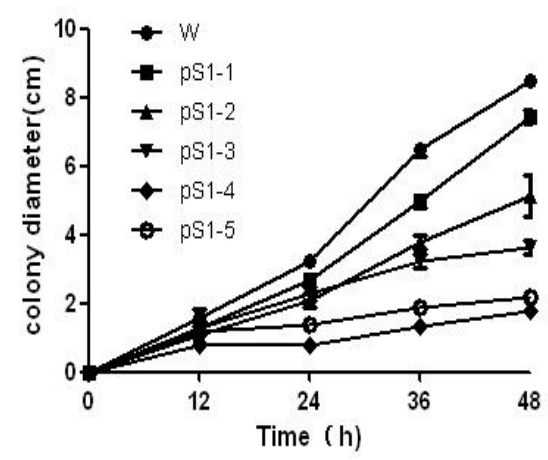

B

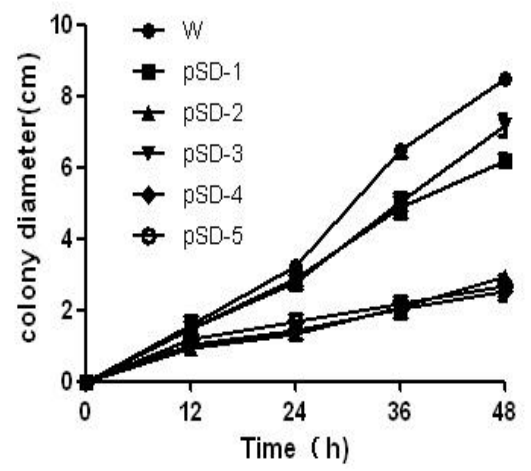

\subsection{SsMADS Essential for Full Pathogenicity}

To determine whether a reduction in $S S M A D S$ expression would confer a change in $S$. sclerotiorum pathogenic capability, we inoculated detached tomato leaves with agar plugs colonized with the wild-type control or with the SSMADS-silencing transformants. When compared with the fully pathogenic wild-type, pathogenicity of the $S S M A D S$-silencing transformants was completely abolished and the transformants pSl-1 and pSD-3 were able to produce disease symptoms (as determined by monitoring the leaves for the appearance of necrotic lesions), but they had fewer disease symptoms than the wild-type strains. Other transformants significantly reduced virulence even 6 days post inoculation (Figure 5A). Transformants pS1-3 and pSD-3 SSMADS expression level lower than wild type-strain, but higher than transformants pS1-2, pS1-4, pS1-5, pSD-2, pSD-4, and pSD-5 (Figure 4C,D), and their pathogenecity also lower than the wild-type strain, but higher than transformants pS1-2, pS1-4, pS1-5 and pSD-2, pSD-4, pSD-5 (Figure 6). These results indicate that silencing of SSMADS expression levels affected S. sclerotiorum virulence.

Figure 6. Pathogenicity of the wild-type and transformant strains: lesion development on tomato leaves. Leaves were inoculated with an agar plug ( $5 \mathrm{~mm}$ in diameter) of each strain. Inoculated leaves were placed in a humidity chamber at $20{ }^{\circ} \mathrm{C}$. Inoculation with the wild-type strain served as the control. Photographs were taken at 6 days after inoculation.

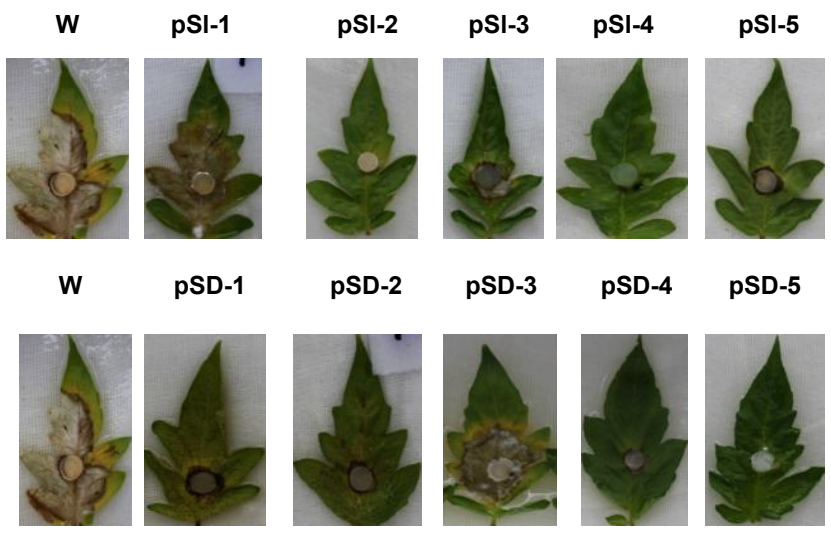




\subsection{Discussion}

The MADS-box proteins are an important family of transcription factors that are involved in the regulation of numerous genes with a diverse range of biological functions. In this study, we describe the cloning and characterization of the $S S M A D S$ gene from the homothallic ascomycete S. sclerotiorum. S. sclerotiorum displays significant similarities to the MADS-box transcription factors from A. oryzae Mcm1, A. nidulans MCMA and M. oryzae MoMCM. MADS box transcription factors are characterized by the MADS-box domain, a conserved amino acid region that is required for DNA binding and dimerization [5]. MADS-box proteins can be classified into the SRF-like (type I) and MEF2-like (type II) classes. Based on phylogenetic analysis and a domain adjacent to the MADS-box domain, the S. sclerotiorum SsMADS protein can be classified as an SRF-like (type I) MADS-box protein. To our knowledge, the SsMADS protein is the first MADS-box transcription factor identified in S. sclerotiorum.

S. cerevisiae contains four MADS-box proteins, Mcm1 and Arg80, which belong to the SRF-like family, and Rlm1 and Smp1, which belong to the MEF2-like family. The MADS-box transcription factor Mcm1 is essential for cell viability and controls $\mathrm{M} / \mathrm{G} 1$ and $\mathrm{G} 2 / \mathrm{M}$ cell-cycle, mating, mini-chromosome maintenance, recombination, and osmotolerance [17-19]. We assessed the growth rate, and our results suggest that $S S M A D S$ plays a major role in aerial hyphal growth. A similar observation was reported in the homothallic ascomycete $S$. macrospora, Fusarium verticillioides, and $M$. oryzae. In $S$. macrospora, the $m c m l$ gene encodes a putative homolog to the $S$. cerevisiae MADS-box protein Mcm1p. The MCM1 protein interacts with the mating-type protein SMTA-1. Deletion of the $S$. macrospora $m \mathrm{~cm} 1$ gene resulted in increased hyphal branching, reduced biomass, and reduced hyphal compartment length during vegetative growth [20,21]. In F. verticillioides, two MADS-box transcription factors, Mads1 and Mads2, were characterized. Compared to the wild-type, the MADS1 and MADS2 knockout mutants exhibited decreased vegetative growth [22]. In M. oryzae, the genome has two putative MADS-box transcription factors, MoMcm1 and Mig1. MoMCM1 encodes a MADS-box protein that is orthologous to $S$. cerevisiae Mcm1, which interacts with both Mst12 and Mata-1 in yeast two-hybrid assays [23,24]. MoMCM1 is required for normal vegetative growth and conidiation, and is involved in surface recognition and normal appressorium formation. However, in contrast to the above findings, in M. oryzae, another MADS-box transcription factor, Mig1, does not play a major role in vegetative growth, cell wall integrity, or conidiation [25]. The Mig1 gene encodes a MADS-box transcription factor homologous to S. cerevisiae Rlm1. Mig1 has been shown to be a downstream target of the Mps1 MAP kinase in the MPS1 pathway. The mig1 deletion mutant had a normal growth rate and formed melanized appressoria, but it was nonpathogenic and failed to infect rice leaves through wounds.

To determine whether a reduction in $S S M A D S$ expression would confer a change in the pathogenic capability of $S$. sclerotiorum, we performed virulence assays where host material was inoculated with hyphae of wild-type or the $S S M A D S$-silencing transformants. The results indicate that silencing of SSMADS expression affected S. sclerotiorum virulence. Studies of the Mcm1 homologues in Sorda macrospora, $M$. oryzea, and F. verticillioides have further demonstrated the role of SRF-type MADS-box proteins in sexual mating and virulence in filamentous fungi [21,26]. In S. macrospora, in addition to the vegetative defects observed, deletion of $\mathrm{mcm} 1$ dramatically affected the sexual fertility 
of $S$. macrospora. The $m c m 1$ deletion mutant was only capable of producing protoperithecia and was unable to form either ascospores or perithecia [20,21]. The M. oryzea migl deletion mutant was nonpathogenic and failed to infect rice leaves through wounds. The Migl gene deletion has no obvious effect on the initial plant penetration processes, but blocks the differentiation of secondary infectious hyphae. Mig1 may be required for overcoming plant defense responses and the differentiation of secondary infectious hyphae in live plant cells [25]. M. oryzea MoMCM1 is important for appressorial penetration and infectious growth, and is required for male fertility and microconidium production. MoMcm1 interacts with Mst12 and MatA-1 to regulate germ tube identity and male fertility, respectively [23]. However, in F. verticillioides, MADS-box MADS1 and MADS2 are not required for pathogenicity. The deletion of MADS1 and MADS2 failed to produce perithecia and ascospores, which affect sexual development. However, deletion of either gene did not have an effect on the ability of F. verticillioides to colonize maize stalk or kernels [22]. Perhaps the mechanism by which $F$. verticillioides invades plant tissue is different from that of $M$. oryzea. F. verticillioides does not have the capability to mechanically penetrate plant cuticles, it does not produce appressorium-like structures, and it can only colonize plant tissue through mechanical or insect damage [26].

\section{Experimental Section}

\subsection{Strains and Plasmids}

The wild-type strain (S. sclerotiorum 1980) and transformants generated from S. sclerotiorum in this study were cultured on potato dextrose agar (PDA) medium at $25^{\circ} \mathrm{C}$ and stored as a pure culture at $-80{ }^{\circ} \mathrm{C}$. The silencing vector pSilent-1 [15] and pSilent-Dual1 [16] were obtained from the Fungal Genetics Stock Center (Kansas City, MO, USA). The RNA-silencing vector pSilent-1 carries a hygromycin resistance cassette and a transcriptional unit for hairpin RNA expression with a spacer of a cutinase gene intron from the rice blast fungus $M$. oryzae. The RNA-silencing vector pSilent-Dual1 carries a geneticin resistance cassette and two convergent opposing RNA polymerase II promoters, PtrpC and Pgpd (from the Aspergillus nidulans trpC promoter and the gpd promoter) for filamentous fungi.

\subsection{Cloning of the SsMADS Gene}

To collect the hyphae from cultures grown on solid PDA medium for 5 days, a sterile cellophane membrane was placed on the medium before inoculation. Total RNA was extracted using the Trizol reagent (TaKaRa Biotechnology Co., Ltd., Dalian, China) according to the manufacturer's instructions. A cDNA synthesis kit (TaKaRa Biotechnology Co., Ltd.) was used according the manufacturer's instructions. All primers used in this study are listed in Table 1. Referring to the GenBank SsMADS transcription factor gene sequence (accession: FJ869956), primers FP1 and RP2 were designed using the software Primer 5.0 (Premier Biosoft International, Palo Alto, CA, USA), and the cloned SsMADS gene cDNA was amplified using polymerase chain reaction (PCR). The PCR-amplified fragments were detected using 1\% agarose gel electrophoresis, and the fragments obtained after the cloning vector pMD18-T was connected and transformed into E. coli DH5a. Using bacterial PCR after digestion, a positive monoclonal was selected and sent to Beijing Invitrogen Corporation (Beijing, China) for sequencing. 
Table 1. Primers used in this study.

\begin{tabular}{cc}
\hline Primer Name & Sequence (5'-3') \\
\hline FP1 & CGAGCTCATGGCCGATATCACAGATCAACACGAC \\
RP2 & CAAGCTTTGATTGATGTGCTTGCGGTTGTGGC \\
pSl-L-1 & CCGCTCGAGGCTCGTGGAATTAAACGCGCAAG (XhoI) \\
pS1-L-2 & CCCAAGCTTGAGCCATATAATTTGGTACGC (HindIII) \\
pS1-R-1 & GGGGTACCGCTCGTGGAATTAAACGCGCAAG $(\mathrm{K} p n \mathrm{I})$ \\
pS1-R-2 & GAAGATCTGAGCCATATAATTTTGGTACGC $(\mathrm{B} g l \mathrm{II})$ \\
SsSrf-1 & CCCTCTAGAGCTCGTGGAATTAAACGCGCAAG $(\mathrm{X} b a \mathrm{I})$ \\
SsSrf-2 & CCCTCTAGAGAGCCATATAATTTGGTACGC $(\mathrm{X} b a \mathrm{I})$ \\
hyg-F & CGACAGCGTCTCCGACCTGA \\
hyg-R & CGCCCAAGCTGCATCATCGAA \\
gen-F & TGTCCGGTGCCCTGAATGAACT \\
gen-R & GCCGCCAAGCTCTTCAGCAATAT \\
kmads11 & GCGTCGCCACATCACATTC \\
kmads12 & TTTCCCTTCCGCCTTTGTG \\
ActF & CCCAGCGTTCTACGTCT \\
ActR & CATGTCAACACGAGCAATG \\
\hline
\end{tabular}

\subsection{Construction of RNAi Reconstructed Plasmids}

To silence the SsMADS gene, $S$. sclerotiorum SsMADS gene cDNA was used as a template. The SsMADS gene cDNA $520 \mathrm{bp}$ fragment was chosen as the target gene, and primers were designed using the software Primer 5.0. The left arm primer fragments were pSl-L-1 (with XhoI restriction sites) and pSl-L-2 (with HindIII restriction sites). The right arm primers pSl-R-1 (with KpnI restriction sites) and pSI-R-2 (digested with BglII sites) were used. A 520-bp length of the SsMADS gene cDNA fragments was cloned. Plasmids derived from pSilent-1 were digested using XhoI, HindIII, KpnI, and BglII enzymes. The reconstructed plasmid pS1-SSMADS was eventually obtained by ligating the digested products into pSilent-1 using $\mathrm{T}_{4}$ ligase. The primer SsSrf-1 and SsSrf-2 was designed with the specific restriction sites $\mathrm{X} b a \mathrm{I}$ and a 520-bp length of the SSMADS gene cDNA fragment was cloned. Plasmids derived from pSilent-Duall, the promoter for the RNA interference plasmid vector, were digested with the same $\mathrm{X} b a \mathrm{I}$ enzymes used to integrate the $S S M A D S$ cDNA fragments. The reconstructed plasmid pSD-SSMADS was eventually obtained by ligating the digested products into pSilent-Duall with $\mathrm{T}_{4}$ ligase. All primers used in this study are listed in Table 1.

\subsection{Preparation of S. sclerotiorum Protoplasts}

The lysing enzyme Novozyme was dissolved in filter-sterilized buffer, which was added to S. sclerotiorum mycelium and shaken at $100 \mathrm{rpm}$ and $28^{\circ} \mathrm{C}$ for $1-3 \mathrm{~h}$. Whole mycelium was lysed by adding $\mathrm{KCl}$ and the lysis was verified using an optical microscope; the mixture was centrifuged, washed, and diluted. Plate count was determined using a hemocytometer. Plasma cell concentration was diluted to $1 \times 10^{8} / \mathrm{mL}$. Protoplasts $(1 \mathrm{~mL})$ were added, along with $12.5 \mu \mathrm{L}$ DMSO, $62.5 \mu \mathrm{L}$ Heparin, 166.7 $\mu \mathrm{L}, 60 \%$ PEG (polyethylene glycol), and $83.3 \mu \mathrm{L} \mathrm{KTC} \mathrm{(1.2} \mathrm{M} \mathrm{KCl,} 10 \mathrm{mM}$ Tris- $\mathrm{HCl}$, $\mathrm{pH} 7.5,50 \mathrm{mM} \mathrm{CaCl}_{2}$ ). Protoplasts were used for transformation. 


\subsection{Transformation of Reconstructed Plasmids into S. sclerotiorum}

S. sclerotiorum protoplasts $(50 \mu \mathrm{L})$ were added to ice-cold reconstructed plasmid pS1-SsMADS or pSD-SSMADS and placed on ice for $30 \mathrm{~min}$. PEG solution $(1 \mathrm{~mL})$ was then added and mixed gently at room temperature for $20 \mathrm{~min}$. RM medium (22.96 g sucrose, $0.05 \mathrm{~g}$ yeast extract, $0.8 \mathrm{~g}$ agar, constant volume $100 \mathrm{~mL}$ ) was coated onto the places and they were cultured at $25{ }^{\circ} \mathrm{C}$ for $15 \mathrm{~h}$ in the dark. Then, $8 \mathrm{~mL}$ of a solution containing $100 \mu \mathrm{g} / \mathrm{mL}$ hygromycin $\mathrm{B}$ or geneticin was added to the $\mathrm{RM}$ medium and cultured at $25^{\circ} \mathrm{C}$ until colonies grew [27].

\subsection{Screening and Analysis for Phenotypic Transformants}

The transformations originated from RNAi reconstructed plasmids transformed from S. sclerotiorum were used for a phenotypic screen. They were inoculated on the PDA media and grown at $25{ }^{\circ} \mathrm{C}$. Representative transformant phenotypes from strains pSl-1, pSl-2, pSl-3, pSl-4, and pSl-5 (from reconstructed plasmid pS1-SsMADS transformations) and pSD-1, pSD-2, pSD-3, pSD-4, and pSD-5 (from reconstructed plasmid pSD-SSMADS transformations) were chosen. Transformants were identified by amplifying hygromycin- or geneticin-resistant gene fragments with their specific primers (hyg-F/hyg-R or gen-F/gen-R) [28]. The PCR reaction was conducted as follows: denaturation at $94{ }^{\circ} \mathrm{C}$ for $5 \mathrm{~min}$, then the cycling parameters were denaturation at $94{ }^{\circ} \mathrm{C}$ for $30 \mathrm{~s}$, annealing at $55{ }^{\circ} \mathrm{C}$ for $30 \mathrm{~s}$, and extension at $72{ }^{\circ} \mathrm{C}$ for $1 \mathrm{~min}$. A total of 32 cycles were performed. The cycles were followed by an extension at $72{ }^{\circ} \mathrm{C}$ for $10 \mathrm{~min}$. The PCR-amplified fragments were detected using $1 \%$ agarose gel electrophoresis.

\subsection{Detection of Growth Rates}

The wild type strain and transforms strains pSl-1, pSl-2, pSl-3, pSl-4, pSl-5, and pSD-1, pSD-2, pSD-3, pSD-4, and pSD-5 cultured on PDA medium for $25^{\circ} \mathrm{C}$ for 3 days. Mycelial outer edge blocks were taken from the colony using a punch (diameter $5 \mathrm{~mm}$ ), and then placed centrally onto PDA plates and placed in an incubator at $25{ }^{\circ} \mathrm{C}$. This was repeated three times for each strain. The block surrounding the colony diameter was measured every $12 \mathrm{~h}$.

\subsection{Real-Time RT-PCR Analysis of SsMADS Gene Expression}

SSMADS transcript expression levels in the wild-type strains and different transformants was evaluated using real-time RT-PCR on an ABI PRISM 7500 system. Transformants and the wild-type strain were cultured on PDA for 5 days. Total RNA from these strains was extracted and single-stranded cDNA was synthesized using a PrimeScript RT reagent Kit (TaKaRa Biotechnology Co., Ltd.). The single-stranded cDNA was used to amplify actin and SsMADS genes using two pairs of primers (kmads11/kmads12 and ActF/ActR, respectively). The PCR reaction was conducted as follows: denaturation at $95{ }^{\circ} \mathrm{C}$ for $30 \mathrm{~s}$, then the cycling parameters were: denaturation at $95{ }^{\circ} \mathrm{C}$ for $5 \mathrm{~s}$, and annealing at $55{ }^{\circ} \mathrm{C}$ for $40 \mathrm{~s}$, for a total of 40 cycles. The cycles were followed by an extension at $72{ }^{\circ} \mathrm{C}$ for $10 \mathrm{~min}$. The expression level of each transcript was calculated using Gel-Pro analyzer 4 software (Media Cybernetics, Rockville, MD, USA) with the actin gene as the internal control. In all 
experiments, samples were amplified in triplicate, and the average cycle threshold was calculated and used to determine the relative expression of each transformant.

\subsection{Pathogenicity Tests}

Four-week-old greenhouse tomato seedlings were used in the infection assay. Fully expanded tomato leaves were excised and placed onto a $15 \mathrm{~cm}$ enamel plate with water-saturated gauze. Individual leaves were inoculated with a single $0.5 \mathrm{~cm}$ mycelium colonized agar plug obtained from the expanding margins of the PDA cultured wild-type or SsMADS transformant strain colonies. Inoculated leaves were placed in a humidity chamber at $20{ }^{\circ} \mathrm{C}$ for 6 days.

\section{Conclusions}

We selected the $S$. sclerotiorum SsMADS MADS-box transcription factor to undergo detailed functional characterization in this study. The putative $S s M A D S$ gene was cloned in our research, and it was highly similar to the orthologues $S$. cerevisiae Mcm1, including a conserved DNA-binding domain. The SSMADS gene belongs to the SRF-like MADS-box proteins. SsMADS function was investigated using RNA interference, and these $S S M A D S$-silenced strains had a significantly reduced growth rate compared with the wild-type strain. Infection assays of the silenced strains on tomato leaflets indicated that $S S M A D S$ was required for pathogenesis on the leaves of a susceptible host. Our results suggest that the MADS-box transcription factor SsMADS is involved in S. sclerotiorum growth and virulence.

\section{Acknowledgments}

This work was supported in part by the National Natural Science Foundation of China (No. 31101394, No. 31271991, and No. 81201268); the Special Program of the Ministry of Agriculture for Public Profession (No. 201103016); The Ministry of Education Fund for the Doctoral of China (No. 20120061110082) and Jilin University Interdisciplinary Innovation Project (No. 450060481141). We are grateful to the Fungal Genetics Stock Center (Kansas City, MO, USA) for providing the plasmids pSilent-1 and pSilent-Dual1.

\section{Author Contributions}

X.Q. and B.Y. performed cloning gene, analyzed data and wrote the paper; J.L., X.Z., and G.L. performed construction plasmids; D.Z., L.L., X.W., and L.W. performed transformation; J.C. and W.M. performed analysis transformants; H.P. and Y.Z. conceived and designed the experiments.

\section{Conflicts of Interest}

The authors declare no conflicts of interest. 


\section{References}

1. Boland, G.J.; Hall, R. Index of plant hosts of Sclerotinia sclerotiorum. Can. J. Plant Pathol. 1994, 16, 93-108.

2. Dong, T.; Hu, Z.; Deng, L.; Wang, Y.; Zhu, M.; Zhang, J.; Chen, G. A tomato MADS-box transcription factor, SIMADS1, acts as a negative regulator of fruit ripening. Plant Physiol. 2013, 163, 1026-1036.

3. Kofuji, R.; Sumikawa, N.; Yamasaki, M.; Kondo, K.; Ueda, K.; Ito, M.; Hasebe, M. Evolution and divergence of the MADS-box gene family based on genome-wide expression analyses. Mol. Biol. Evol. 2003, 20, 1963-1977.

4. Messenguy, F.; Dubois, E. Role of MADS box proteins and their cofactors in combinatorial control of gene expression and cell development. Gene 2003, 316, 1-21.

5. Shore, P.; Sharrocks, A.D. The MADS-box family of transcription factors. Eur. J. Biochem. 1995, 229, 1-13.

6. Parenicova, L.; de Folter, S.; Kieffer, M.; Horner, D.S.; Favalli, C.; Busscher, J.; Cook, H.E.; Ingram, R.M.; Kater, M.M.; Davies, B.; et al. Molecular and phylogenetic analyses of the complete MADS-box transcription factor family in Arabidopsis: New openings to the MADS world. Plant Cell 2003, 15, 1538-1551.

7. Liu, Y.; Cui, S.; Wu, F.; Yan, S.; Lin, X.; Du, X.; Chong, K.; Schilling, S.; Theissen, G.; Meng, Z. Functional conservation of MIKC-Type MADS box genes in Arabidopsis and rice pollen maturation. Plant Cell 2013, 25, 1288-1303.

8. Perilleux, C.; Pieltain, A.; Jacquemin, G.; Bouche, F.; Detry, N.; D’Aloia, M.; Thiry, L.; Aljochim, P.; Delansnay, M.; Mathieu, A.S.; et al. A root chicory MADS box sequence and the Arabidopsis flowering repressor FLC share common features that suggest conserved function in vernalization and de-vernalization responses. Plant J. 2013, 75, 390-402.

9. Uddenberg, D.; Reimegard, J.; Clapham, D.; Almqvist, C.; von Arnold, S.; Emanuelsson, O.; Sundstrom, J.F. Early cone setting in Picea abies acrocona is associated with increased transcriptional activity of a MADS box transcription factor. Plant Physiol. 2013, 161, 813-823.

10. Treisman, R.; Ammerer, G. The SRF and MCM1 transcription factors. Curr. Opin. Genet. Dev. 1992, 2, 221-226.

11. Wang, D.; Chang, P.S.; Wang, Z.; Sutherland, L.; Richardson, J.A.; Small, E.; Krieg, P.A.; Olson, E.N. Activation of cardiac gene expression by myocardin, a transcriptional cofactor for serum response factor. Cell 2001, 105, 851-862.

12. Puri, P.L.; Wu, Z.; Zhang, P.; Wood, L.D.; Bhakta, K.S.; Han, J.; Feramisco, J.R.; Karin, M.; Wang, J.Y. Induction of terminal differentiation by constitutive activation of p38 MAP kinase in human rhabdomyosarcoma cells. Genes Dev. 2000, 14, 574-584.

13. Sclerotinia sclerotiorum MADS Box Transcription Factor Gene, Complete CDS. Available online: http://www.ncbi.nlm.nih.gov/nucleotide/\%20226938410?report=genbank\&log\$=nucltop\&blast_ rank=1\&rid=gd6eafn5014 (accessed on 22 April 2009).

14. MADS Box Transcription Factor [Sclerotinia sclerotiorum]. Available online: http://www.ncbi. nlm.nih.gov/protein/226938411 (accessed on 22 April 2009). 
15. Nakayashiki, H.; Hanada, S.; Nguyen, B.Q.; Kadotani, N.; Tosa, Y.; Mayama, S. RNA silencing as a tool for exploring gene function in ascomycete fungi. Fungal Genet. Biol. 2005, 42, 275-283.

16. Nguyen, Q.B.; Kadotani, N.; Kasahara, S.; Tosa, Y.; Mayama, S.; Nakayashiki, H. Systematic functional analysis of calcium-signalling proteins in the genome of the rice-blast fungus, Magnaporthe oryzae, using a high-throughput RNA-silencing system. Mol. Microbiol. 2008, 68, 1348-1365.

17. Alvarez-Buylla, E.R.; Pelaz, S.; Liljegren, S.J.; Gold, S.E.; Burgeff, C.; Ditta, G.S.; de Pouplana, L.R.; Martinez-Castilla, L.; Yanofsky, M.F. An ancestral MADS-box gene duplication occurred before the divergence of plants and animals. Proc. Natl. Acad. Sci. USA 2000, 97, 5328-5333.

18. Elble, R.; Tye, B.K. Chromosome loss, hyperrecombination, and cell cycle arrest in a yeast mcm1 mutant. Mol. Biol. Cell 1992, 3, 971-980.

19. McInerny, C.J.; Partridge, J.F.; Mikesell, G.E.; Creemer, D.P.; Breeden, L.L. A novel Mcm1-dependent element in the SWI4, CLN3, CDC6, and CDC47 promoters activates M/G1-specific transcription. Genes Dev. 1997, 11, 1277-1288.

20. Nolting, N.; Poggeler, S. A MADS box protein interacts with a mating-type protein and is required for fruiting body development in the homothallic ascomycete Sordaria macrospora. Eukaryot. Cell 2006, 5, 1043-1056.

21. Nolting, N.; Poggeler, S. A STE12 homologue of the homothallic ascomycete Sordaria macrospora interacts with the MADS box protein MCM1 and is required for ascosporogenesis. Mol. Microbiol. 2006, 62, 853-868.

22. Ortiz, C.S.; Shim, W.B. The role of MADS-box transcription factors in secondary metabolism and sexual development in the maize pathogen Fusarium verticillioides. Microbiology 2013, 159, 2259-2268.

23. Zhou, X.; Liu, W.; Wang, C.; Xu, Q.; Wang, Y.; Ding, S.; Xu, J.R. A MADS-box transcription factor MoMcml is required for male fertility, microconidium production and virulence in Magnaporthe oryzae. Mol. Microbiol. 2011, 80, 33-53.

24. Park, G.; Xue, C.; Zheng, L.; Lam, S.; Xu, J.R. MST12 regulates infectious growth but not appressorium formation in the rice blast fungus Magnaporthe grisea. Mol. Plant Microbe Interact. 2002, 15, 183-192.

25. Mehrabi, R.; Ding, S.; Xu, J.R. MADS-box transcription factor mig1 is required for infectious growth in Magnaporthe grisea. Eukaryot. Cell 2008, 7, 791-799.

26. Duncan, K.E.; Howard, R.J. Biology of maize kernel infection by Fusarium verticillioides. Mol. Plant Microbe Interact. 2010, 23, 6-16.

27. Rollins, J.A. The Sclerotinia sclerotiorum pacl gene is required for sclerotial development and virulence. Mol. Plant Microbe Interact. 2003, 16, 785-795.

28. Zhang, Y.; Li, G.; He, D.; Yu, B.; Yokoyama, K.; Wang, L. Efficient insertional mutagenesis system for the dimorphic pathogenic fungus Sporothrix schenckii using Agrobacterium tumefaciens. J. Microbiol. Methods 2011, 84, 418-422.

(C) 2014 by the authors; licensee MDPI, Basel, Switzerland. This article is an open access article distributed under the terms and conditions of the Creative Commons Attribution license (http://creativecommons.org/licenses/by/3.0/). 